\title{
SIGN CHANGING SOLUTIONS FOR ELLIPTIC EQUATIONS WITH CRITICAL GROWTH IN CYLINDER TYPE DOMAINS*
}

\author{
Pedro Girẽo ${ }^{1}$ And Miguel Ramos ${ }^{2}$
}

\begin{abstract}
We prove the existence of positive and of nodal solutions for $-\Delta u=|u|^{p-2} u+\mu|u|^{q-2} u$, $u \in \mathrm{H}_{0}^{1}(\Omega)$, where $\mu>0$ and $2<q<p=2 N(N-2)$, for a class of open subsets $\Omega$ of $\mathbb{R}^{N}$ lying between two infinite cylinders.
\end{abstract}

Mathematics Subject Classification. 35J20, 35J25, 35J65, 35B05.

Received December 20, 2001.

\section{INTRODUCTION}

We are concerned with the existence of nonzero solutions for the nonlinear second order elliptic equation

$$
-\Delta u=|u|^{p-2} u+\mu|u|^{q-2} u, \quad u \in \mathrm{H}_{0}^{1}(\Omega)
$$

where $\Omega$ is a smooth unbounded domain of $\mathbb{R}^{N}$ with $N \geq 3, \mu \in \mathbb{R}^{+}, 2<q<p$ and $p$ is the critical Sobolev exponent $p=2^{*}=2 N /(N-2)$. Without loss of generality we assume that $0 \in \Omega$.

In the case where $\Omega$ is bounded, the proof of the existence of positive and of nodal (sign changing) solutions for $(\mathrm{P})$ or similar equations goes back to the work in $[3,4,10]$. In the case where $\Omega$ is unbounded and $p$ is subcritical $\left(p<2^{*}\right)$, we refer for example to [5,12]. On the other hand, motivated by the work in $[1,2,5,7]$, in [8] the authors prove the existence of a positive solution for a class of unbounded domains, concerning the (somewhat simpler) equation $-\Delta u=\lambda u+|u|^{p-2} u$, where $\lambda$ is positive and small (see also [9] for a related result).

The present work complements the quoted results. Following [5,8], we fix a number $1 \leq \ell \leq N-1$ and write $\mathbb{R}^{N}=\mathbb{R}^{\ell} \times \mathbb{R}^{N-\ell}, z=(t, y) \in \mathbb{R}^{\ell} \times \mathbb{R}^{N-\ell}$. For a given subset $A \subset \mathbb{R}^{N-\ell}$ we denote $\bar{A}_{\delta}=\left\{y \in \mathbb{R}^{N-\ell}\right.$ : $\operatorname{dist}(y, A)<\delta\}$ and $\widehat{A}=\mathbb{R}^{\ell} \times A$. Also, for $t \in \mathbb{R}^{\ell}$ we let $\Omega^{t}=\left\{y \in \mathbb{R}^{N-\ell}:(t, y) \in \Omega\right\}$. We shall consider both situations $(\mathrm{H})$ and $(\mathrm{H})_{0}$ below:

(H) there exist two nonempty bounded open sets $F \subset G \subset \mathbb{R}^{N-\ell}$ such that $F$ is a Lipschitz domain and $\widehat{F} \subset \Omega \subset \widehat{G}$. Moreover, for each $\delta>0$ there is $R>0$ such that $\Omega^{t} \subset F_{\delta}$ for all $|t| \geq R$;

Keywords and phrases: Nodal solutions, cylindrical domains, semilinear elliptic equation, critical Sobolev exponent, concentration-compactness.

* The authors are partially supported by FCT.

1 Mathematics Department, IST, Av. Rovisco Pais, 1049-001 Lisboa, Portugal; e-mail: girao@math.ist.utl.pt

2 CMAF and Faculty of Sciences, Universidade de Lisboa, Av. Prof. Gama Pinto, 2, 1649-003 Lisboa, Portugal; e-mail: mramos@lmc.fc.ul.pt 
$(\mathrm{H})_{0}$ there exists an open bounded set $G \subset \mathbb{R}^{N-\ell}$ such that $\Omega \subset \widehat{G}$ and moreover for each $\delta>0$ there is $R>0$ such that $\Omega^{t} \subset B_{\mathbb{R}^{N-\ell}}(0, \delta)$ for all $|t| \geq R$.

We have denoted by $B_{\mathbb{R}^{N-\ell}}(0, \delta)$ the open ball in $\mathbb{R}^{N-\ell}$ centered at the origin with radius $\delta>0$. The case $(\mathrm{H})_{0}$ can be seen as a limit case of $(\mathrm{H})$, with $F=\{0\}$. We prove the following:

Theorem 1. Consider problem $(\mathrm{P})$ with $2<q<p=2^{*}$ and assume either $(\mathrm{H})$ or $(\mathrm{H})_{0}$. Then, for every $\mu>0$, the problem admits a positive (and a negative) solution of least energy.

In order to prove the existence of nodal solutions in case $(\mathrm{H})$, we impose further restrictions on $\Omega$, namely that $\Omega$ approaches $\widehat{F}$ "smoothly and slowly".

$(\mathrm{H})^{\prime}$ Assume $(\mathrm{H})$ and that $\Omega$ is of class $\mathrm{C}^{1,1}$ in such a way that the local charts as well as their inverses have uniformly bounded Lipschitz constants. Moreover, there exist constants $m>0$ and $0<a_{1}<a_{0}$ such that $\left(1+\frac{a}{|t|^{m}}\right) F \subset \Omega^{t}$ for every $a \in\left[a_{1}, a_{0}\right]$ and every $|t|$ large.

Theorem 2. Consider problem $(\mathrm{P})$ with $2<q<p=2^{*}$ and assume either $(\mathrm{H})^{\prime}$ or $(\mathrm{H})_{0}$. In case $(\mathrm{H})_{0}$ holds, assume moreover that $q>(N+2) /(N-2)$. Then, for every $\mu>0$, the problem admits a sign changing solution.

In Theorem 2 the conclusion is that $(\mathrm{P})$ has a pair of sign changing solutions, since the nonlinearity is odd. In case $(\mathrm{H})_{0}$, the extra restriction on $q$ is merely needed in lower dimensions $(N=3,4,5)$, since $(N+2) /(N-2) \geq 2$ for $N \geq 6$. In fact, Theorem 2 still holds if $q=(N+2) /(N-2)$ provided $\mu$ is sufficiently large (see the remark which follows the proof of Prop. 2.5).

The proof of our main theorems is given in Section 2 (see Props. 1.1 and 1.4); it relies on the concentrationcompactness principle at infinity and on some ideas of $[4,8]$. Section 3 provides technical estimates which are needed in the proof of Theorem 2. We also give further information on the decay properties of the solutions found in Theorems 1 and 2.

\section{Concentration-Compactness}

It is well known that the solutions of $(\mathrm{P})$ correspond to critical points of the energy functional (for simplicity of notations, we take $\mu=1$ in $(\mathrm{P}))$ :

$$
I(u)=\frac{1}{2} \int|\nabla u|^{2}-\frac{1}{p} \int|u|^{p}-\frac{1}{q} \int|u|^{q}, \quad u \in \mathrm{H}_{0}^{1}(\Omega),
$$

where the integrals are taken over the domain $\Omega$. We recall $2<q<p=2^{*}$. It follows from assumptions $(\mathrm{H})$ or $(\mathrm{H})_{0}$ that we can choose the norm $\|u\|:=\left(\int|\nabla u|^{2}\right)^{1 / 2}$ in $\mathrm{H}_{0}^{1}(\Omega)$. Let

$$
c_{0}:=\inf \left\{I(u): u \in \mathrm{H}_{0}^{1}(\Omega), u \neq 0 \text { and } I^{\prime}(u) u=0\right\} .
$$

It is also clear that $c_{0}>0$ and that every nonzero critical point $u$ of $I$ is such that $I(u) \geq c_{0}$. The following result proves Theorem 1 .

Proposition 1.1. Under assumptions $(\mathrm{H})$ or $(\mathrm{H})_{0}$, the infimum in $(1.1)$ is attained in a critical point of $I$.

Proof. 1. We shall omit what concerns standard arguments $(c f .[3,4])$. We first recall that there exists a Palais-Smale sequence $\left(u_{n}\right) \subset \mathrm{H}_{0}^{1}(\Omega)$ at level $c_{0}$, namely

$$
I\left(u_{n}\right) \rightarrow c_{0} \quad \text { and } \quad I^{\prime}\left(u_{n}\right) \rightarrow 0 .
$$

Since moreover $c_{0}>0$, equation (1.2) implies that liminf $\left\|u_{n}\right\|>0$. This sequence is bounded and, up to a subsequence, $u_{n} \rightarrow u$ weakly in $\mathrm{H}_{0}^{1}(\Omega), u_{n}(x) \rightarrow u(x)$ a.e. and $I^{\prime}(u)=0, I(u) \geq 0$. Since $\liminf \left\|u_{n}\right\|>0$ and 
$I^{\prime}\left(u_{n}\right) u_{n} \rightarrow 0$, we also have that $\liminf \int\left|u_{n}\right|^{p}>0$; indeed, if $\int\left|u_{n}\right|^{p} \rightarrow 0$ along a subsequence, then, since $\left(\int u_{n}^{2}\right)$ is bounded, by interpolation $\int\left|u_{n}\right|^{q} \rightarrow 0$, whence $\left\|u_{n}\right\| \rightarrow 0$, as $I^{\prime}\left(u_{n}\right) u_{n} \rightarrow 0$.

2. Up to subsequences, there exist measures $\mu$ and $\nu$ on $\Omega$ such that $\left|\nabla\left(u_{n}-u\right)\right|^{2} \rightarrow \mu$ and $\left|u_{n}-u\right|^{p} \rightarrow \nu$ weakly in the space $M(\Omega)$ of finite measures in $\Omega$. Clearly, $\|\mu\| \geq S\|\nu\|^{2 / p}$, where $S$ is the best constant for the embedding $\mathrm{H}^{1}\left(\mathbb{R}^{N}\right) \subset \mathrm{L}^{\mathrm{p}}\left(\mathbb{R}^{N}\right)$. By testing $I^{\prime}\left(u_{n}\right) \rightarrow 0$ with $u_{n} \varphi$ for any $\varphi \in \mathcal{D}\left(\mathbb{R}^{N}\right)$ and since $I^{\prime}(u) u \varphi=0$ we also see that

$$
\|\mu\|=\|\nu\| .
$$

In particular,

$$
\mu \neq 0 \Rightarrow\|\mu\| \geq S^{p /(p-2)}=S^{N / 2}
$$

3. Define

$$
\begin{aligned}
\mu_{\infty} & :=\lim _{R \rightarrow \infty} \limsup _{n \rightarrow \infty} \int_{|x|>R}\left|\nabla u_{n}\right|^{2}, \\
\nu_{\infty} & :=\lim _{R \rightarrow \infty} \limsup _{n \rightarrow \infty} \int_{|x|>R}\left|u_{n}\right|^{p} \\
\eta_{\infty} & :=\lim _{R \rightarrow \infty} \limsup _{n \rightarrow \infty} \int_{|x|>R}\left|u_{n}\right|^{q} .
\end{aligned}
$$

Again, it is clear that

$$
\mu_{\infty} \geq S \nu_{\infty}^{2 / p}
$$

By testing $I^{\prime}\left(u_{n}\right) \rightarrow 0$ with $u_{n} \psi_{R}(R>0)$ where $\psi_{R} \in \mathrm{C}^{\infty}(\Omega), 0 \leq \psi_{R} \leq 1$ is such that $\psi_{R}(x)=0$ if $|x| \leq R$ and $\psi_{R}(x)=1$ if $|x| \geq R+1$, it follows easily that

$$
\mu_{\infty}=\nu_{\infty}+\eta_{\infty}
$$

4. We recall from $[1,2,11]$ that

$$
\begin{aligned}
\int\left|\nabla u_{n}\right|^{2} & =\int|\nabla u|^{2}+\|\mu\|+\mu_{\infty}+\mathrm{o}(1), \\
\int\left|u_{n}\right|^{p} & =\int|u|^{p}+\|\nu\|+\nu_{\infty}+\mathrm{o}(1), \\
\int\left|u_{n}\right|^{q} & =\int|u|^{q}+\eta_{\infty}+\mathrm{o}(1) .
\end{aligned}
$$

As a consequence, and thanks to $(1.2,1.3)$ and $(1.6)$, we have that

$$
c_{0}=I(u)+\left(\frac{1}{2}-\frac{1}{p}\right)\|\mu\|+\left(\frac{1}{2}-\frac{1}{p}\right) \nu_{\infty}+\left(\frac{1}{2}-\frac{1}{q}\right) \eta_{\infty}
$$

In particular, $c_{0} \geq I(u)$. Since $I^{\prime}(u)=0$, the proof will be complete once we show that $u \neq 0$. Indeed, in this case we have that $I(u) \geq c_{0}$, whence $I(u)=c_{0}$. (Incidentally, Eqs. (1.6) and (1.7) also show that, in fact, $\|\mu\|=\mu_{\infty}=0$, hence $u_{n} \rightarrow u$ in $\mathrm{H}_{0}^{1}(\Omega)$.) 
5. We recall from [3] that $c_{0}<S^{N / 2} / N$. Since (1.7) implies that

$$
c_{0} \geq\left(\frac{1}{2}-\frac{1}{p}\right)\|\mu\|=\frac{1}{N}\|\mu\|,
$$

we deduce from $(1.3,1.4)$ that $\mu=\nu=0$. Thus $u_{n} \rightarrow u$ in $\mathrm{H}_{\mathrm{loc}}^{1}(\Omega)$ and

$$
c_{0}=I(u)+\left(\frac{1}{2}-\frac{1}{p}\right) \nu_{\infty}+\left(\frac{1}{2}-\frac{1}{q}\right) \eta_{\infty} .
$$

6. Suppose first that $\Omega=\widehat{F}$. Since $\liminf \int\left|u_{n}\right|^{p}>0$, by Lemma 2.1 in [8] we may assume that, up to translations, $\int_{B_{1}(0)}\left|u_{n}\right|^{p} \geq c$ for some $c>0$. Since $u_{n} \rightarrow u$ in $\mathrm{H}_{\mathrm{loc}}^{1}(\Omega)$, we conclude that $u \neq 0$ and this proves Proposition 1.1 for the case $\Omega=\widehat{F}$. Moreover, the argument shows that $c_{0}\left(\widehat{F_{\delta}}\right) \rightarrow c_{0}(\widehat{F})$ as $\delta \rightarrow 0$ (see $(\mathrm{H})$ and (1.12) for the notations).

7. We complete the proof in case $(\mathrm{H})_{0}$ holds. Assume by contradiction that $u=0$. Then, clearly $\int u_{n}^{2} \rightarrow 0$ (see e.g. (2.1) in [8]). By interpolation, also $\int\left|u_{n}\right|^{q} \rightarrow 0$. In particular, $\eta_{\infty}=0$. Since $c_{0}<S^{N / 2} / N$, equations (1.5, 1.6) and (1.8) show that then $\nu_{\infty}=0$, whence, by the second identity in Step $4, \int\left|u_{n}\right|^{p} \rightarrow 0$. This contradicts the fact that $\lim \inf \int\left|u_{n}\right|^{p}>0$ and proves Proposition 1.1 under $(\mathrm{H})_{0}$.

8. At last, we consider the case where $(\mathrm{H})$ holds and $\Omega \neq \widehat{F}$. Again, assume by contradiction that $u=0$. Let $\delta>0$ be given and take $R>0$ according to assumption (H). Let $\psi_{R}$ be as in Step 3 and denote

$$
v_{n}=u_{n} \psi_{R} \in \mathrm{H}_{0}^{1}\left(\widehat{F_{\delta}}\right) .
$$

Since $u_{n} \rightarrow 0$ in $\mathrm{H}_{\mathrm{loc}}^{1}(\Omega)$, clearly we have that

$$
I\left(v_{n}\right)=I\left(u_{n}\right)+\mathrm{o}(1) \quad \text { and } \quad I^{\prime}\left(v_{n}\right) v_{n}=\mathrm{o}(1) .
$$

We claim that

$$
I\left(v_{n}\right)+\mathrm{o}(1) \geq c_{0}\left(\widehat{F_{\delta}}\right) .
$$

Assuming the claim for a moment, it follows from $(1.9,1.10)$ that

$$
c_{0}=I\left(u_{n}\right)+\mathrm{o}(1)=I\left(v_{n}\right)+\mathrm{o}(1) \geq c_{0}\left(\widehat{F_{\delta}}\right) .
$$

Since $\delta>0$ is arbitrary, we conclude that $c_{0} \geq c_{0}(\widehat{F})$. On the other hand, since $\widehat{F} \subset \Omega$ and $c_{0}(\widehat{F})$ is attained (see Step 6 above), we must have that $c_{0}<c_{0}(\widehat{F})$. This contradiction completes the proof.

It remains to prove the inequality in (1.10). For this, we observe that (1.9) together with the fact that $\liminf I\left(u_{n}\right)>0$ implies that $\liminf \left\|v_{n}\right\|>0$ and $\liminf \int\left|v_{n}\right|^{p}>0$. Now, let

$$
w_{n}=t_{n} v_{n} \quad\left(t_{n}>0\right)
$$

be such that $I^{\prime}\left(w_{n}\right) w_{n}=0$; namely, $t_{n}$ is given by

$$
\frac{t_{n}^{p-2} \int\left|v_{n}\right|^{p}+t_{n}^{q-2} \int\left|v_{n}\right|^{q}}{\int\left|\nabla v_{n}\right|^{2}}=1
$$


Then $\left(t_{n}\right)$ is bounded and, since $I^{\prime}\left(v_{n}\right) v_{n} \rightarrow 0$, we see that $t_{n} \rightarrow 1$. In particular,

$$
I\left(w_{n}\right)=I\left(v_{n}\right)+\mathrm{o}(1)
$$

Now, by definition, $I\left(w_{n}\right) \geq c_{0}\left(\widehat{F_{\delta}}\right)$ and (1.10) follows from (1.11).

Using the notation in assumption $(\mathrm{H})$, we denote

$$
c_{0}(\widehat{F}):=\inf \left\{I(u): u \in \mathrm{H}_{0}^{1}(\widehat{F}), u \neq 0 \text { and } I^{\prime}(u) u=0\right\}<S^{N / 2} / N .
$$

We also let

$$
c_{0}^{\infty}:=c_{0}(\widehat{F}) \quad \text { in case }(\mathrm{H}), \quad c_{0}^{\infty}:=S^{N / 2} / N \quad \text { in case }(\mathrm{H})_{0}
$$

We have shown in the proof of Proposition 1.1 that $c_{0}(\widehat{F})$ is attained by a critical point of the energy functional in $\mathrm{H}_{0}^{1}(\widehat{F})$. In fact, the argument above yields the following compactness result.

Proposition 1.2. Under assumptions $(\mathrm{H})$ or $(\mathrm{H})_{0}$, let $\left(u_{n}\right) \subset \mathrm{H}_{0}^{1}(\Omega)$ be such that

$$
\limsup I\left(u_{n}\right)<c_{0}^{\infty} \quad \text { and } \quad I^{\prime}\left(u_{n}\right)\left(u_{n} \psi\right) \rightarrow 0
$$

for every $\psi \in \mathrm{C}^{\infty}(\Omega) \cap W^{1, \infty}(\Omega)$. Suppose $u_{n} \rightarrow u$ weakly in $\mathrm{H}_{0}^{1}(\Omega), u_{n}(x) \rightarrow u(x)$ a.e. and $I^{\prime}(u)(u \psi)=0$ for such functions $\psi$. Then $u_{n} \rightarrow u$ in $\mathrm{H}_{0}^{1}(\Omega)$.

Proof. Since $I^{\prime}(u) u=0$, we have that $I(u) \geq 0$. Denote $v_{n}:=u_{n}-u$. By the Brezis-Lieb lemma,

$$
I\left(v_{n}\right)=I\left(u_{n}\right)-I(u)+\mathrm{o}(1)<c_{0}^{\infty}+\mathrm{o}(1)
$$

and

$$
I^{\prime}\left(v_{n}\right)\left(v_{n} \psi\right)=I^{\prime}\left(u_{n}\right)\left(u_{n} \psi\right)-I^{\prime}(u)(u \psi)+\mathrm{o}(1) \rightarrow 0
$$

for every $\psi \in \mathrm{C}^{\infty}(\Omega) \cap W^{1, \infty}(\Omega)$. Since $\left(v_{n}\right)$ converges weakly to zero, a similar (though easier) argument as in the proof of Proposition 1.1 shows that we cannot have $\lim \sup I\left(v_{n}\right)>0$. Thus $I\left(v_{n}\right) \rightarrow 0$. Since also $I^{\prime}\left(v_{n}\right) v_{n} \rightarrow 0$, we conclude that $\left\|v_{n}\right\| \rightarrow 0$, hence $u_{n} \rightarrow u$ in $\mathrm{H}_{0}^{1}(\Omega)$.

Next we turn to the proof of Theorem 2. Following [4], let

$$
c_{1}:=\inf \left\{I(u): u \in \mathrm{H}_{0}^{1}(\Omega), u^{ \pm} \neq 0 \text { and } I^{\prime}\left(u^{ \pm}\right) u^{ \pm}=0\right\} \geq c_{0}>0
$$

where we denote $u^{+}=\max \{u, 0\}$ and $u^{-}=\max \{-u, 0\}$. The following proposition will be proved in Section 3 (cf. Props. 2.4 and 2.5).

Proposition 1.3. Assume $(\mathrm{H})^{\prime}$ or $(\mathrm{H})_{0}$ holds; in the latter case, we also assume that $q>(N+2) /(N-2)$. Then

$$
c_{1}<c_{0}+c_{0}^{\infty}
$$

Our final result completes the proof of Theorem 2.

Proposition 1.4. Assume $(\mathrm{H})^{\prime}$ or $(\mathrm{H})_{0}$ holds; in the latter case, we also assume that $q>(N+2) /(N-2)$. Then the infimum in (1.15) is attained in a critical point of $I$.

Proof. It is known (cf. [4]) that there exists a Palais-Smale sequence at level $c_{1}$, namely

$$
I\left(u_{n}\right) \rightarrow c_{1} \quad \text { and } \quad I^{\prime}\left(u_{n}\right) \rightarrow 0
$$


with the additional property that

$$
I\left(u_{n}^{ \pm}\right) \geq c_{0}+\mathrm{o}(1)
$$

(so that, in fact, $\left.c_{1} \geq 2 c_{0}\right)$. As in Step 1 in the proof of Proposition 1.1, modulo a subsequence, $\left(u_{n}\right)$ converges weakly in $\mathrm{H}_{0}^{1}(\Omega)$ and pointwise a.e. to a critical point $u$ of $I$. Observe that $I^{\prime}\left(u_{n}\right) \rightarrow 0$ implies that

$$
I^{\prime}\left(u_{n}^{ \pm}\right)\left(u_{n}^{ \pm} \psi\right)=I^{\prime}\left(u_{n}\right)\left(u_{n}^{ \pm} \psi\right) \rightarrow 0
$$

for every $\psi \in \mathrm{C}^{\infty}(\Omega) \cap W^{1, \infty}(\Omega)$. Similarly, $I^{\prime}\left(u^{ \pm}\right)\left(u^{ \pm} \psi\right)=0$. Since moreover $I\left(u_{n}\right)=I\left(u_{n}^{+}\right)+I\left(u_{n}^{-}\right)=c_{1}+\mathrm{o}(1)$, we deduce from (1.16) and Proposition 1.3 that

$$
\lim \sup I\left(u_{n}^{ \pm}\right)<c_{0}^{\infty} .
$$

It follows from $(1.17,1.18)$ and Proposition 1.2 that $u_{n}^{ \pm} \rightarrow u^{ \pm}$in $\mathrm{H}_{0}^{1}(\Omega)$. Hence $u_{n} \rightarrow u$ in $\mathrm{H}_{0}^{1}(\Omega), I(u)=c_{1}$ and $I\left(u^{ \pm}\right) \geq c_{0}>0$. This finishes the proof.

\section{DeCAy AND ENERgy EStimates}

This section is devoted to general equations of the form

$$
-\Delta u-\lambda u=g(u), \quad u \in \mathrm{H}_{0}^{1}(\Omega)
$$

where $\Omega \subset \mathbb{R}^{N}(N \geq 3)$ is an open set with $\mathrm{C}^{1,1}$ boundary and $g$ satisfies (recall that $p=2^{*}=2 N /(N-2)$ )

$$
|g(s)| \leq C\left(|s|+|s|^{p-1}\right), \quad \forall s \in \mathbb{R} .
$$

Under assumption (2.2), it follows from the Brezis-Kato estimates and classical elliptic regularity theory that the solutions of $(2.1)$ lie in $\mathrm{C}^{2}(\Omega) \cap \mathrm{L}^{\infty}(\Omega) \cap \mathrm{C}(\bar{\Omega})$. In view of the applications that we have in mind (cf. assumptions $\left.(\mathrm{H})-(\mathrm{H})_{0}\right)$, we let $\mathbb{R}^{N}=\mathbb{R}^{\ell} \times \mathbb{R}^{N-\ell}$ with $1 \leq \ell<N$ and accordingly write $(t, y) \in \mathbb{R}^{\ell} \times \mathbb{R}^{N-\ell}$ for any point $(t, y) \in \mathbb{R}^{N}$.

Proposition 2.1. Let $\Omega=\mathbb{R}^{\ell} \times F$ where $F \subset \mathbb{R}^{N-\ell}$ is a $\mathrm{C}^{1,1}$ domain and let $g \in \mathrm{C}^{1}(\mathbb{R})$ satisfy $(2.2), g(0)=0$ and $g^{\prime}(s)=o\left(s^{\varepsilon}\right)$ near 0 , for some $\varepsilon>0$. Let $u$ be a solution of

$$
-\Delta u-\lambda u=g(u), \quad u \in \mathrm{H}_{0}^{1}(\Omega),
$$

where $\lambda<\lambda_{1}$ and $\lambda_{1}$ is the first eigenvalue of $\left(-\Delta, \mathrm{H}_{0}^{1}(F)\right)$. Then

$$
|u(t, y)|+\left|\nabla_{t} u(t, y)\right| \leq \varphi(y) \mathrm{e}^{-\sqrt{1+\left(\lambda_{1}-\lambda\right)|t|^{2}}}, \quad \forall(t, y) \in \Omega,
$$

where $\varphi$ is a positive eigenfunction associated to $\lambda_{1}$. Also, there exists a constant $C>0$ such that

$$
|\nabla u(t, y)| \leq C \mathrm{e}^{-\sqrt{1+\left(\lambda_{1}-\lambda\right)|t|^{2}}}, \quad \forall(t, y) \in \Omega .
$$

Proof. 1. Since $u \in \mathrm{L}^{\infty}(\Omega)$, we have from (2.2) that $|g(u(x))| \leq c|u(x)|$ for every $x \in \Omega$. By elliptic regularity theory (Th. 9.15 of [6]), there exists $c>0$ such that, for all $\alpha \geq 2$,

$$
\|u\|_{W^{2, \alpha}\left(B_{1}(0) \times F\right)} \leq c\|u\|_{L^{\alpha}\left(B_{2}(0) \times F\right)} .
$$


Due to invariance by translations,

$$
\|u\|_{W^{2, \alpha}\left(B_{1}(t) \times F\right)} \leq c\|u\|_{\mathrm{L}^{\alpha}\left(B_{2}(t) \times F\right)} \quad \forall t \in \mathbb{R}^{\ell} .
$$

In particular,

$$
u(t, y) \rightarrow 0 \text { as }|t| \rightarrow+\infty, \text { uniformly for } y \in F
$$

and

$$
|\nabla u(t, y)| \rightarrow 0 \text { as }|t| \rightarrow+\infty, \text { uniformly for } y \in F \text {. }
$$

2. Suppose $\mu \in] \lambda, \lambda_{1}[$ is fixed and let

$$
\Psi(t):=\alpha \mathrm{e}^{-\sqrt{1+\left(\lambda_{1}-\mu\right)|t|^{2}}} \in \mathrm{H}^{1}\left(\mathbb{R}^{\ell}\right),
$$

where $\alpha$ will be chosen later. An easy computation shows that

$$
-\Delta \Psi+\left(\lambda_{1}-\mu\right) \Psi=\left(\lambda_{1}-\mu\right) \Psi\left((\ell-1) \theta^{-1 / 2}+\theta^{-1}+\theta^{-3 / 2}\right)
$$

where $\theta(t):=1+\left(\lambda_{1}-\mu\right)|t|^{2}$. In particular,

$$
-\Delta \Psi+\left(\lambda_{1}-\mu\right) \Psi \geq \frac{\alpha\left(\lambda_{1}-\mu\right)}{1+\left(\lambda_{1}-\mu\right)|t|^{2}} \mathrm{e}^{-\sqrt{1+\left(\lambda_{1}-\mu\right)|t|^{2}}}=: h(t) .
$$

Let $\varphi$ be a positive eigenfunction associated to $\lambda_{1}$ and

$$
z(t, y):=\varphi(y) \Psi(t)
$$

The function $z$ satisfies

$$
-\Delta z-\mu z \geq \varphi(y) h(t)
$$

Hence, for $w:=z-u$, we have

$$
-\Delta w-\mu w \geq \varphi(y) h(t)+(\mu-\lambda) u-g(u)=: k(t, y) .
$$

Since $g(0)=0=g^{\prime}(0)$, it follows from (2.6) that if $u(t, y) \geq 0$, then

$$
(\mu-\lambda) u-g(u) \geq 0
$$

if $|t|>R$, where $R$ is chosen large; hence also $k(t, y) \geq 0$. In summary,

$$
w<0 \Rightarrow-\Delta w-\mu w \geq 0
$$

if $|t|>R$. Since $\partial z / \partial \nu=h \partial \varphi / \partial \nu<0$ ( $\nu$ stands for the outward normal to $\partial \Omega$ ), we can fix $\alpha$ so large that $w \geq 0$ for $|t| \leq R$. Let $\omega:=\{x \in \Omega: w(x)<0\}$. Since

$$
w^{-}(x)=0 \quad \forall x \in \partial \omega
$$


by multiplying (2.9) by $w^{-}$and integrating, it follows from (2.10) that $\omega=\emptyset$. Therefore $u \leq z$. In the same way we can prove that $-u \leq z$, and so

$$
|u(t, y)| \leq \varphi(y) \mathrm{e}^{-\sqrt{1+\left(\lambda_{1}-\mu\right)|t|^{2}}}, \quad \forall(t, y) \in \Omega ;
$$

the constant $\alpha$ has been incorporated into the function $\varphi$.

3. We now improve the previous estimate. Since $g^{\prime}(s)=o\left(s^{\varepsilon}\right)$, there exists $C>0$ such that

$$
|g(u(t, y))| \leq C|u(t, y)|^{1+\varepsilon}, \quad \forall(t, y) \in \Omega .
$$

We fix $\mu \in] \lambda, \lambda_{1}[$, sufficiently close to $\lambda$, so that

$$
\gamma:=(1+\varepsilon) \sqrt{\lambda_{1}-\mu}>\sqrt{\lambda_{1}-\lambda}
$$

Combining (2.11) and (2.12),

$$
|g(u(t, y))| \leq C \varphi(y)^{1+\varepsilon} \mathrm{e}^{-\gamma|t|}, \quad \forall(t, y) \in \Omega .
$$

Let $z(t, y):=\varphi(y) \Psi(t)$, where $\Psi$ is like in Step 2, with $\mu$ replaced by $\lambda$. For $w:=z-u$, we have

$$
-\Delta w-\lambda w \geq \frac{\alpha\left(\lambda_{1}-\lambda\right)}{1+\left(\lambda_{1}-\lambda\right)|t|^{2}} \varphi(y) \mathrm{e}^{-\sqrt{1+\left(\lambda_{1}-\lambda\right)|t|^{2}}}-g(u(t, y))=: p(t, y) .
$$

Since $\gamma>\sqrt{\lambda_{1}-\lambda}$, it follows from (2.13) that $p(t, y) \geq 0$ if $|t|$ is large. Choosing $\alpha$ sufficiently large leads to $p \geq 0$ in $\Omega$. We conclude from the maximum principle, as before, that $u \leq z$ in $\Omega$ and in the same way, $|u| \leq z$ in $\Omega$.

4. To finish the proof we use the decay of $u$. Specifically, the derivatives $v=\partial u / \partial t_{i}$, for $i=1, \ldots, \ell$, satisfy

$$
-\Delta v-\lambda v=g^{\prime}(u) v \quad \text { and } v \in \mathrm{H}_{0}^{1}(\Omega) .
$$

The argument in Steps 2 and 3 above proves an analogous decay for $v$. The main point in the final argument is that if $\mu \in] \lambda, \lambda_{1}$ [ is sufficiently close to $\lambda$ then

$$
\frac{\alpha\left(\lambda_{1}-\lambda\right)}{1+\left(\lambda_{1}-\lambda\right)|t|^{2}} \varphi(y) \mathrm{e}^{-\sqrt{1+\left(\lambda_{1}-\lambda\right)|t|^{2}}}-C \varphi^{\varepsilon}(y) \mathrm{e}^{-\varepsilon \sqrt{1+\left(\lambda_{1}-\lambda\right)|t|^{2}}} \times \varphi(y) \mathrm{e}^{-\sqrt{1+\left(\lambda_{1}-\mu\right)|t|^{2}}}
$$

is positive for $|t|$ large. The final assertion in the statement of Proposition 2.1 follows from (2.5).

We now consider the setting analyzed in Section 2. Again, we denote by $\lambda_{1}=\lambda_{1}(F)$ the first eigenvalue of $\left(-\Delta, \mathrm{H}_{0}^{1}(F)\right)$.

Proposition 2.2. Suppose $\Omega$ is a domain satisfying assumption $(\mathrm{H})$ and moreover that $\Omega$ is of class $\mathrm{C}^{1,1}$ in such a way that the local charts as well as their inverses have uniformly bounded Lipschitz constants. Let $g \in \mathrm{C}^{1}(\mathbb{R})$ be as in Proposition 2.1 and $u$ be a solution of

$$
-\Delta u-\lambda u=g(u), \quad u \in \mathrm{H}_{0}^{1}(\Omega),
$$

with $\lambda<\lambda_{1}$. Then, for each $\left.\bar{\lambda} \in\right] \lambda, \lambda_{1}[$, there exists a constant $C>0$ such that

$$
|u(t, y)|+|\nabla u(t, y)| \leq C \mathrm{e}^{-\sqrt{1+(\bar{\lambda}-\lambda)|t|^{2}}}, \quad \forall(t, y) \in \Omega .
$$


Proof. The proof is similar to that of Proposition 2.1, so we just stress the differences. Thanks to our assumption on $\Omega$, the constant $c$ in $(2.5)$ can be taken uniformly bounded, hence (2.6) still holds. Now, fix $\delta>0$ in such a way that $\lambda<\lambda_{1}\left(F_{\delta}\right)<\lambda_{1}$. Running through the argument in Step 2 of the proof of Proposition 2.1 we see that, similarly to $(2.11)$,

$$
|u(t, y)| \leq \varphi(y) \mathrm{e}^{-\sqrt{1+\left(\lambda_{1}\left(F_{\delta}\right)-\mu\right)|t|^{2}}}, \quad \forall(t, y) \in \Omega,|t| \geq R
$$

provided $R>0$ is sufficiently large; here, $\mu \in] \lambda, \lambda_{1}\left(F_{\delta}\right)\left[\right.$ and $\varphi$ is an eigenfunction associated to $\lambda_{1}\left(F_{\delta}\right)$. Arguing as in Step 3 of the quoted proof, the previous estimate for $u$ can be improved to

$$
|u(t, y)| \leq \varphi(y) \mathrm{e}^{-\sqrt{1+\left(\lambda_{1}\left(F_{\delta}\right)-\lambda\right)|t|^{2}}}, \quad \forall(t, y) \in \Omega,|t| \geq R
$$

This clearly implies that we can choose $C>0$ such that

$$
|u(t, y)| \leq C \mathrm{e}^{-\sqrt{1+\left(\lambda_{1}\left(F_{\delta}\right)-\lambda\right)|t|^{2}}}, \quad \forall(t, y) \in \Omega
$$

A similar decay estimate for the derivatives of $u$ follows from (2.5) and (2.14). Since $\lambda_{1}\left(F_{\delta}\right)$ can be chosen arbitrarily close to $\lambda_{1}$ (see Lem. 2.3 of [8]), this proves the proposition.

Going back to Proposition 2.1, it may be interesting to observe that the asymptotic estimates can be sharpened as follows:

Proposition 2.3. Under the assumptions of Proposition 2.1, let $u$ be a solution of problem (2.3). Then:

(a) the conclusion of Proposition 2.1 still holds with $\mathrm{e}^{-\sqrt{1+\left(\lambda_{1}-\lambda\right)|t|^{2}}}$ replaced by $\mathrm{e}^{-\sqrt{1+\left(\lambda_{1}-\lambda\right)|t|^{2}}}|t|^{-\frac{\ell-1}{2}}$;

(b) (Hopf lemma) If $u$ is positive and $\eta<\lambda$ then $u(t, y) \geq \widetilde{\varphi}(y) \mathrm{e}^{-\sqrt{1+\left(\lambda_{1}-\eta\right)|t|^{2}}}$ for every $(t, y) \in \Omega$, for some positive eigenfunction $\widetilde{\varphi}$ associated to $\lambda_{1}$.

Proof. (a) We improve the estimate (2.4) by repeating the argument with

$$
\Psi(t):=\mathrm{e}^{-\sqrt{1+\left(\lambda_{1}-\lambda\right)|t|^{2}}}|t|^{-\frac{\ell-1}{2}} .
$$

Indeed,

$$
-\Delta \Psi+\left(\lambda_{1}-\lambda\right) \Psi=\Psi\left(\left(\lambda_{1}-\lambda\right) \theta^{-1}+\left(\lambda_{1}-\lambda\right) \theta^{-3 / 2}+\frac{\ell-1}{2} \frac{\ell-3}{2} \frac{1}{|t|^{2}}\right),
$$

a computation that can be easily checked using (2.8); here, of course, $\theta(t):=1+\left(\lambda_{1}-\lambda\right)|t|^{2}$. As a consequence, for sufficiently large $|t|$ we have that

$$
-\Delta \Psi+\left(\lambda_{1}-\lambda\right) \Psi \geq \frac{1}{2} \mathrm{e}^{-\sqrt{1+\left(\lambda_{1}-\lambda\right)|t|^{2}}}|t|^{-\frac{\ell+3}{2}}=: h(t)
$$

Due to the assumptions on $g$, for the function on $w:=\alpha \varphi \Psi-u$, with $\alpha$ a fixed positive number, we have

$$
-\Delta w-\lambda w \geq \alpha h(t) \varphi(y)-A \varphi^{1+\varepsilon}(y) \mathrm{e}^{-(1+\varepsilon) \sqrt{1+\left(\lambda_{1}-\lambda\right)|t|^{2}}} .
$$

The right hand member above is positive for sufficiently large $|t|$. Using the maximum principle, we conclude, as in (2.11), that

$$
|u(t, y)| \leq \alpha \varphi(y) \mathrm{e}^{-\sqrt{1+\left(\lambda_{1}-\lambda\right)|t|^{2}}}|t|^{-\frac{\ell-1}{2}}, \quad \forall(t, y) \in \Omega
$$


Finally, as in Step 4 of the quoted proof, a similar estimate for the derivatives of $u$ follows from $(2.4,2.15)$ and the fact that

$$
\frac{\alpha}{2} \varphi(y) \mathrm{e}^{-\sqrt{1+\left(\lambda_{1}-\lambda\right)|t|^{2}}}|t|^{-\frac{\ell+3}{2}}-C \varphi^{\varepsilon}(y) \mathrm{e}^{-\varepsilon \sqrt{1+\left(\lambda_{1}-\lambda\right)|t|^{2}}}|t|^{-\varepsilon \frac{\ell-1}{2}} \times \varphi(y) \mathrm{e}^{-\sqrt{1+\left(\lambda_{1}-\lambda\right)|t|^{2}}}
$$

is positive for $|t|$ large.

(b) Here we let $\Psi(t):=\mathrm{e}^{-\sqrt{1+\left(\lambda_{1}-\eta\right)|t|^{2}}}$. Fix any $\left.\mu \in\right] \eta, \lambda[$. Similarly to (2.8), we can check that

$$
h(t):=-\Delta \Psi+\left(\lambda_{1}-\mu\right) \Psi \leq 0 \quad \text { for every }|t| \geq R
$$

with $R$ sufficiently large. Since $u(t, y) \rightarrow 0$ as $|t| \rightarrow \infty$ and since $g(0)=0=g^{\prime}(0)$ we can choose $R$ in such a way that also $(\mu-\lambda) u-g(u) \leq 0$ for $|t| \geq R$. Letting $z:=\varphi \Psi$, we can fix a small $\alpha>0$ so that $w:=\alpha z-u \leq 0$ if $|t| \leq R$; this is possible because $u \in \mathrm{C}^{1}(\bar{\Omega}), u>0$ in $\Omega$ and $\partial u / \partial \nu<0$ on $\partial \Omega$ (outward normal derivative). In summary, we have that (compare with $(2.9)$ )

$$
-\Delta w-\mu w=\alpha \varphi h+(\mu-\lambda) u-g(u)=: k(t, y)
$$

and $k(t, y) \leq 0$ for $|t| \geq R$, while $w \leq 0$ for $|t| \leq R$. Using the maximum principle as in the proof of Proposition 2.1 we conclude that $w \leq 0$ for all $(t, y)$.

We end this section with the proof of Proposition 1.3, which is contained in Propositions 2.4 and 2.5 below. We will refer to the functional $I$ introduced at the beginning of Section 2 as well as to the quantities $c_{0}, c_{0}^{\infty}$ and $c_{1}$ defined in $(1.1,1.13)$ and $(1.15)$, respectively.

Proposition 2.4. Assume $(\mathrm{H})^{\prime}$ holds. Then $c_{1}<c_{0}+c_{0}^{\infty}$.

Proof. 1. We know that $c_{0}$ is attained by a positive function $v \in \mathrm{H}_{0}^{1}(\Omega)$ and $c_{0}^{\infty}$ is attained by some positive function $\psi \in \mathrm{H}_{0}^{1}\left(\mathbb{R}^{\ell} \times F\right)$ (cf. Prop. 1.1). Let $m>0$ and $0<a_{1}<a_{0}$ be given by assumption $(\mathrm{H})^{\prime}$ and denote $A:=a_{0} / a_{1}>1$. Fix a large number $M$ such that $M>2 A$ and

$$
\frac{a_{1}}{a_{0}}<\left(\frac{M-A}{M+A}\right)^{m}
$$

Let $\rho: \mathbb{R} \rightarrow \mathbb{R}$ be a smooth function such that $\rho(s)=1$ for $|s| \leq 1$ and $\rho(s)=0$ for $|s| \geq A$. We define $\rho_{R}$ and $\eta_{R}$ in $\mathbb{R}^{\ell}$ by $\rho_{R}=\rho(|t| / R)$ and $\eta_{R}(t)=\rho_{R}\left(t-M R \mathbf{e}_{1}\right)=\rho\left(\left|\frac{t}{R}-M \mathbf{e}_{1}\right|\right)$. We also let

$$
v_{R}(t, y):=v(t, y) \rho_{R}(t)
$$

and

where

$$
\psi_{R}(t, y):=\lambda_{R}^{-N / p} \psi\left(\frac{t-M R \mathbf{e}_{1}}{\lambda_{R}}, \frac{y}{\lambda_{R}}\right) \eta_{R}(t)
$$

$$
\lambda_{R}:=1+\frac{a_{0}}{(M+A)^{m} R^{m}} .
$$

We observe that $v_{R}$ and $\psi_{R}$ have disjoint supports. Moreover, both functions belong to $\mathrm{H}_{0}^{1}(\Omega)$ if $R$ is sufficiently large. Indeed, suppose $(t, y) \in \partial \Omega$ and let us show that $\psi_{R}(t, y)=0$. We may already assume that $\left|t-M R \mathbf{e}_{1}\right|$ $\leq A R$. In particular,

$$
(M-A) R \leq|t| \leq(M+A) R .
$$


Now, to prove the claim it is sufficient to show that $\left(\frac{t-M R \mathbf{e}_{1}}{\lambda_{R}}, \frac{y}{\lambda_{R}}\right) \notin \widehat{F}$, i.e. that $\frac{y}{\lambda_{R}} \notin F$. Observing that

$$
y=\left(1+\frac{a}{|t|^{m}}\right) \frac{y}{\lambda_{R}}
$$

where, according to $(2.16-2.18)$,

$$
a:=a_{0}\left(\frac{|t|}{(M+A) R}\right)^{m} \in\left[a_{1}, a_{0}\right],
$$

the conclusion follows from $(\mathrm{H})^{\prime}$ and the fact that $(t, y) \notin \Omega$.

2. Thanks to Proposition 2.2 (with $\lambda=0$ ), we know that $|v(t, y)|+|\nabla v(t, y)|=\mathrm{O}\left(\mathrm{e}^{-\delta|t|}\right)$ and similarly for $\psi$. Here and henceforth $\delta$ denotes various positive constants. It then follows easily that $I\left(v_{R}\right) \rightarrow I(v)$ and $I\left(\psi_{R}\right) \rightarrow I(\psi)$ as $R \rightarrow \infty$ and also that

$$
I\left(v_{R}\right)=I(v)+\mathrm{O}\left(\mathrm{e}^{-\delta R}\right), \quad I\left(\psi_{R}\right)=I(\psi)+\mathrm{O}\left(\mathrm{e}^{-\delta R}\right)
$$

In fact, the second estimate can be improved, observing that

$$
\int \psi_{R}^{p}=\int \psi^{p} \rho_{R}^{p}=\int \psi^{p}+\int \psi^{p}\left(\rho_{R}^{p}-1\right)=\int \psi^{p}+\mathrm{O}\left(\mathrm{e}^{-\delta R}\right)
$$

and similarly $\int\left|\nabla \psi_{R}\right|^{2}=\int|\nabla \psi|^{2}+\mathrm{O}\left(\mathrm{e}^{-\delta R}\right)$, while

$$
\int \psi_{R}^{q}=\lambda_{R}^{N\left(1-\frac{q}{p}\right)} \int \psi^{q}+\mathrm{O}\left(\mathrm{e}^{-\delta R}\right)
$$

so that

$$
\begin{aligned}
I\left(\psi_{R}\right) & =I(\psi)+\left(1-\lambda_{R}^{N\left(1-\frac{q}{p}\right)}\right) \int \psi^{q}+\mathrm{O}\left(\mathrm{e}^{-\delta R}\right) \\
& \leq I(\psi)-N\left(1-\frac{q}{p}\right) \frac{a_{0}}{(M+A)^{m} R^{m}} \int \psi^{q}+\mathrm{O}\left(\mathrm{e}^{-\delta R}\right),
\end{aligned}
$$

whence, for every sufficiently large $R$,

$$
I\left(\psi_{R}\right)<I(\psi)
$$

3. Clearly, as in $(2.19,2.20)$, for large $R$ and uniformly for $\tau_{1}, \tau_{2} \in[1 / 2,2]$, we have that

$$
\begin{aligned}
I\left(\tau_{1} v_{R}-\tau_{2} \psi_{R}\right) & =I\left(\tau_{1} v_{R}\right)+I\left(\tau_{2} \psi_{R}\right)<I\left(\tau_{1} v\right)+I\left(\tau_{2} \psi\right) \\
& \leq \sup _{s \geq 0} I(s v)+\sup _{s \geq 0} I(s \psi)=c_{0}+c_{0}^{\infty} .
\end{aligned}
$$

The last equality above is a direct consequence of the definitions of $c_{0}$ and $c_{0}^{\infty}$, by standard arguments (cf. $[3,4,11])$. In summary, there exists $R_{0}$ such that

$$
\sup _{1 / 2 \leq \tau_{1}, \tau_{2} \leq 2} I\left(\tau_{1} v_{R}-\tau_{2} \psi_{R}\right)<c_{0}+c_{0}^{\infty}, \quad \forall R \geq R_{0}
$$

4. Thanks to $(2.21)$, to complete the proof it remains to show that there exist $\tau_{1}, \tau_{2} \in[1 / 2,2]$ and $R \geq R_{0}$ such that $w:=\tau_{1} v_{R}-\tau_{2} \psi_{R}$ satisfies $I^{\prime}\left(w^{ \pm}\right) w^{ \pm}=0$. Since $v_{R}$ and $\psi_{R}$ have disjoint supports, this amounts to prove 
that there exist $\tau_{1}, \tau_{2} \in[1 / 2,2]$ and $R \geq R_{0}$ such that

$$
I^{\prime}\left(\tau_{1} v_{R}\right) v_{R}=0 \quad \text { and } \quad I^{\prime}\left(\tau_{2} \psi_{R}\right) \psi_{R}=0 .
$$

Now, we have that $I^{\prime}\left(v_{R} / 2\right) v_{R} \rightarrow I^{\prime}(v / 2) v>0$ and $I^{\prime}\left(2 v_{R}\right) v_{R} \rightarrow I^{\prime}(2 v) v<0$ as $R \rightarrow \infty$ and similarly for $\psi$. Hence (2.22) follows by applying the intermediate value theorem.

Proposition 2.5. Assume $(\mathrm{H})_{0}$ holds and moreover that $q>(N+2) /(N-2)$. Then $c_{1}<c_{0}+c_{0}^{\infty}=c_{0}+S^{N / 2} / N$. Proof. Let $U(x)=c_{N} /\left(1+|x|^{2}\right)^{(N-2) / 2}$ be the Talenti instanton, normalized in such a way that $\int|U|^{p}=$ $\int|\nabla U|^{2}=S^{N / 2}$ (i.e. $\left.c_{N}=(N(N-2))^{(N-2) / 4}\right)$. Let $U_{\varepsilon}(x)=\varepsilon^{-N / p} U(x / \varepsilon)$ be its rescaling, so that also $\int\left|U_{\varepsilon}\right|^{p}=\int\left|\nabla U_{\varepsilon}\right|^{2}=S^{N / 2}$. The following argument is similar to that in [12], except that we cut down the least energy solution and also $U_{\varepsilon}$ and estimate the error in doing so, instead of computing the interference between their energies.

Recall that, without loss of generality, we are assuming that $0 \in \Omega$. By Proposition 1.1, we know that $c_{0}$ is achieved by a positive function $v \in \mathrm{H}_{0}^{1}(\Omega) \cap \mathrm{C}^{1}(\Omega)$. Let $\rho, \eta: \mathbb{R} \rightarrow \mathbb{R}$ be smooth functions such that $\rho(s)=1$ for $|s| \leq 1, \rho(s)=0$ for $|s| \geq 2, \eta(s)=0$ for $|s| \leq 2$ and $\eta(s)=1$ for $|s| \geq 3$. We define $\rho_{\varepsilon}$ and $\eta_{\varepsilon}: \mathbb{R}^{N} \rightarrow \mathbb{R}$ by $\rho_{\varepsilon}(x)=\rho(|x| / \sqrt{\varepsilon})$ and $\eta_{\varepsilon}(x)=\eta(|x| / \sqrt{\varepsilon})$. We also define

$$
u_{\varepsilon}:=U_{\varepsilon} \rho_{\varepsilon} \quad \text { and } \quad v_{\varepsilon}:=v \eta_{\varepsilon} .
$$

It is clear that $u_{\varepsilon}$ and $v_{\varepsilon}$ have disjoint supports and that they both belong to $\mathrm{H}_{0}^{1}(\Omega)$. We can estimate

$$
\begin{aligned}
\int\left|\nabla v_{\varepsilon}\right|^{2} & \leq \int|\nabla v|^{2}+2\left(\int_{2 \varepsilon^{1 / 2} \leq|x| \leq 3 \varepsilon^{1 / 2}}\left(|\nabla v|^{2} \eta_{\varepsilon}^{2}+v^{2}\left|\nabla \eta_{\varepsilon}\right|^{2}\right)\right) \\
& \leq \int|\nabla v|^{2}+\mathrm{O}\left(\varepsilon^{N / 2}\right)+\mathrm{O}\left(\varepsilon^{(N-2) / 2}\right) \\
& =\int|\nabla v|^{2}+\mathrm{O}\left(\varepsilon^{(N-2) / 2}\right),
\end{aligned}
$$

while

and similarly for $\int v_{\varepsilon}^{q}$, so that

$$
\int v_{\varepsilon}^{p}=\int v^{p}+\int v^{p}\left(\eta_{\varepsilon}^{p}-1\right) \geq \int v^{p}-\int_{|x| \leq 3 \varepsilon^{1 / 2}} v^{p} \geq \int v^{p}+\mathrm{O}\left(\varepsilon^{N / 2}\right)
$$

$$
I\left(v_{\varepsilon}\right) \leq I(v)+\mathrm{O}\left(\varepsilon^{(N-2) / 2}\right)
$$

As for $u_{\varepsilon}$,

$$
\begin{aligned}
\int\left|\nabla u_{\varepsilon}\right|^{2} & \leq \int\left|\nabla U_{\varepsilon}\right|^{2}+2\left(\int\left|\nabla U_{\varepsilon}\right|^{2} \rho_{\varepsilon}^{2}+U_{\varepsilon}^{2}\left|\nabla \rho_{\varepsilon}\right|^{2}\right) \\
& \leq S^{N / 2}+\mathrm{O}\left(\varepsilon^{(N-2) / 2}\right),
\end{aligned}
$$

while, denoting by $c>0$ some constant which is independent of $\varepsilon$,

$$
\int u_{\varepsilon}^{p} \geq S^{N / 2}+\mathrm{O}\left(\varepsilon^{N / 2}\right) \quad \text { and } \quad \int u_{\varepsilon}^{q} \geq c \varepsilon^{N\left(1-\frac{q}{p}\right)}
$$

as can be checked directly, using the explicit expression of $U_{\varepsilon}$. In summary,

$$
I\left(u_{\varepsilon}\right) \leq\left(\frac{1}{2}-\frac{1}{p}\right) S^{N / 2}+\mathrm{O}\left(\varepsilon^{\frac{N-2}{2}}\right)-c \varepsilon^{N\left(1-\frac{q}{p}\right)} .
$$


Combining (2.23) and (2.24) yields

$$
I\left(u_{\varepsilon}\right)+I\left(v_{\varepsilon}\right) \leq c_{0}+\frac{S^{N / 2}}{N}+c_{1} \varepsilon^{\frac{N-2}{2}}-c_{2} \varepsilon^{N\left(1-\frac{q}{p}\right)},
$$

for some positive constants $c_{1}$ and $c_{2}$. In particular,

$$
I\left(u_{\varepsilon}\right)+I\left(v_{\varepsilon}\right)<c_{0}+\frac{S^{N / 2}}{N}
$$

if $\varepsilon$ is sufficiently small since, by assumption, $\frac{N-2}{2}>N\left(1-\frac{q}{p}\right)$; indeed, this condition is equivalent to $q>p-1=(N+2) /(N-2)$. From (2.26) we can end the proof of Proposition 2.5 with similar arguments as in Steps 3 and 4 in the proof of Proposition 2.4.

Remark 2.6. As observed at the beginning of Section 2, for simplicity of notations we have assumed that $\mu=1$ in problem $(\mathrm{P})$. In the general case, $(2.25)$ reads as

$$
I\left(u_{\varepsilon}\right)+I\left(v_{\varepsilon}\right) \leq c_{0}+\frac{S^{N / 2}}{N}+c_{1} \varepsilon^{\frac{N-2}{2}}-\mu c_{2} \varepsilon^{N\left(1-\frac{q}{p}\right)} .
$$

Thus one still has $(2.26)$ in case $q=(N+2) /(N-2)$ provided $\mu$ is sufficiently large.

\section{REFERENCES}

[1] A.K. Ben-Naoum, C. Troestler and M. Willem, Extrema problems with critical Sobolev exponents on unbounded domains. Nonlinear Anal. TMA 26 (1996) 823-833.

[2] G. Bianchi, J. Chabrowski and A. Szulkin, On symmetric solutions of an elliptic equation with a nonlinearity involving critical Sobolev exponent. Nonlinear Anal. TMA 25 (1995) 41-59.

[3] H. Brezis and L. Nirenberg, Positive solutions of nonlinear elliptic equations involving critical Sobolev exponents. Comm. Pure Appl. Math. 36 (1983) 437-476.

[4] G. Cerami, S. Solimini and M. Struwe, Some existence results for superlinear elliptic boundary value problems involving critical exponents. J. Funct. Anal. 69 (1986) 289-306.

[5] M. Del Pino and P. Felmer, Least energy solutions for elliptic equations in unbounded domains. Proc. Roy. Soc. Edinburgh Sect. A 126 (1996) 195-208.

[6] D. Gilbarg and N.S. Trudinger, Elliptic partial differential equations of second order, Second Edition. Springer, New York, Grundlehren Math. Wiss. 224 (1983).

[7] P.-L. Lions, The concentration-compactness principle in the Calculus of Variations. The limit case, Part 2. Rev. Mat. Iberoamericana 1 (1985) 45-121.

[8] M. Ramos, Z.-Q. Wang and M. Willem, Positive solutions for elliptic equations with critical growth in unbounded domains, in Calculus of Variations and Differential Equations, edited by A. Ioffe, S. Reich and I. Shafrir. Chapman \& Hall/CRC, Boca Raton, FL, Res. Notes in Math. Ser. 140 (2000) 192-199.

[9] I. Schindler and K. Tintarev, Abstract concentration compactness and elliptic equations on unbounded domains, in Prog. Nonlinear Differential Equations Appl., Vol. 43, edited by M.R. Grossinho, M. Ramos, C. Rebelo and L. Sanchez. Birkhäuser, Boston (2001) 369-380.

[10] G. Tarantello, Nodal solutions of semilinear elliptic equations with critical exponent. Differential Integral Equations 5 (1992) $25-42$.

[11] M. Willem, Minimax theorems, in Prog. Nonlinear Differential Equations Appl., Vol. 24. Birkhäuser, Boston (1996).

[12] X.-P. Zhu, Multiple entire solutions of a semilinear elliptic equations. Nonlinear Anal. TMA 12 (1998) 1297-1316. 